\title{
INTERTEXTEXTUALITÉ ET ÉCRITE: L'APPORT DE TEXTE LITTERAIRE DANS UNE EXPERIENCE DE PRODUCTION ÉCRITE AVEC DES LYCEENS AU MAROC
}

\author{
INTERTEXTUALIDADE E ESCRITA: O APORTE DO TEXTO LITERARIO NUMA EXPERIENCIA DE \\ PRODUÇÃO TEXTUAL COM ESTUDANTES DO MARROCOS
}

\author{
Salah Tigourdi ${ }^{1}$ \\ ${ }^{1}$ Universidade Mohammed V (UM5), Rabat, Maroc; Aljahid High School, Safi, Maroc \\ Alliance Française de Safi, Maroc \\ s.tigourdi@gmail.com
}

Reçu le 25 nov. 2019

Accepté le 15 déc. 2019

Résumé: La didactique de la production écrite a profité de la recherche dans différents domaines en mettant en valeur les renouvellements susceptibles d'améliorer cette compétence. II serait convenable de dresser les passerelles entre les notions de l'analyse du discours, notamment l'intertextualité, et la didactique de la production écrite. Dans cet article, nous voulons démontrer l'intérêt du texte littéraire dans les séances de production écrite. Pour ce faire nous avons mené une expérience dans laquelle les apprenants seront amenés à produire des textes narratifs de genre autobiographique. Nous nous sommes appuyés sur la caractéristique de l'intertextualité (G. GENETTE, 1982), les concepts-clés du modèle de Hayes et Flower, en particulier l'environnement de la tâche et la mémoire, ainsi que sur les recommandations didactiques conçues par Jean-Pierre Cuq, incitant les enseignants à l'emploi du texte littéraire dans les activités langagières sur lesquelles s'articulent les différentes compétences du français - en l'occurrence, la production écrite. L'expérience, comme le démontrent ses résultats, illustre la pertinente contribution que peut présenter l'exploitation du texte littéraire comme un modèle de départ dans les activités de production écrite. Les apprenants se veulent motivés puisqu'ils peuvent puiser des éléments narratifs présentés par le texte-support et ont fait preuve d'amélioration de leurs productions écrites.

Mots-clés: Production écrite. Intertextualité. Texte littéraire.

Resumo: A didática da produção escrita se beneficiou da pesquisa em vários campos, destacando as renovações que provavelmente melhorarão essa competência. Seria apropriado construir pontes entre as noções de análise de discurso, especialmente a intertextualidade, e a didática da produção escrita. Neste artigo, desejamos demonstrar a contribuição do texto literário nas atividades de produção escrita. Para fazer isso, realizamos um experimento no qual os alunos são solicitados a produzir textos narrativos autobiográficos. Contamos com a característica da intertextualidade (G.GENETTE, 1982), os conceitos-chave do modelo de Hayes e Flower, em particular o ambiente da tarefa e a memória, bem como as recomendações didáticas feitas por Jean-Pierre Cuq, que incentiva os professores a usar o texto literário em atividades linguísticas nas quais articulam as várias habilidades do francês - neste caso, a produção escrita. A experiência, como demonstram os resultados, ilustra a relevante contribuição que a exploração do texto literário pode oferecer como ponto de partida nas atividades de produção escrita. Os alunos sentiram-se motivados porque puderam tomar como suporte os elementos narrativos apresentados pelo texto-base e demonstraram o aperfeiçoamento de suas produções escritas.

Palavras-chave: Produção escrita. Intertextualidade. Texto literário. 


\section{INTRODUCTION}

Dans les lycées du Maroc, le retour de la littérature française se renforce actuellement une nouvelle loi-cadre qui octroie à la langue française un statut avancé tantôt comme langue d'enseignement pour les DNL (disciplines non-linguistiques) tantôt comme langue enseignée. Cette dernière, aux différents niveaux du secondaire qualifiant, s'articule sur l'enseignement-apprentissage des quatre compétences. A savoir ; compréhension de l'écrit et de l'oral et production de l'oral et de l'écrit qui affronte des obstacles d'ordre épistémologique et didactique. La production écrite, quant à elle, représente l'une des facultés incontournables et constitue la matière évaluable pour justifier le niveau désiré au niveau du lycée. Néanmoins, que ce soit pour les enseignants ou les apprenants, le constat est flagrant: on a de grandes difficultés à produire à l'écrit. Nous voulons par cet article interroger le rôle que peut jouer la littérature dans l'enseignement de la production écrite: comment peut-on intégrer la littérature dans les activités de production écrite ? Après la présentation du fondement théorique de ce travail. Nous mènerons une expérience d'évaluation à la fin de laquelle nous terminerons par une discussion ainsi qu'une conclusion.

\section{LE CADRE THÉORIQUE}

Primo, appuyés sur des recherches linguistiques, neuropsychologiques et connexionnistes, différents auteurs se sont intéressés à la compétence de production de l'écrit (ELLIS, 1988; MARGOLIN, 1984; DE BEAUGRANDE, 1984; MARTLEW, 1983; VAN GALEN, 1991; DELL, 1988; HARLEY, 1984; HOLYOAK, 1991; GARRETT, 1988; LEVELT, 1992;). Nous constatons que les apprenants sont à même de produire des textes de genres autobiographiques. Ce constat a pour fondement théorique le modèle établi par Hayes et Flower (1980) qui se sont appuyés sur une démarche empirique visant à identifier les mécanismes auquel les scripteurs font appel dans la rédaction de textes. Nous voulons par la présente contribution examiner l'apport de deux composantes de ce modèle, à savoir:

D'abord, l'environnement de la tâche (Voir la Fig. 1, qui dresse le schéma du modèle). En effet, cette composante est constituée de toutes les spécificités relatives à la tâche. D'une part, celle-ci comprend les thématiques abordées, l'énonciataire 
ainsi que la motivation que le thème entraine. D'autre part, le texte qui a été produit en amont en ce qu'il se veut objet exogène dans le processus scriptural.

Ensuite, la mémoire à long terme du scripteur dans laquelle il puise toutes les connaissances, métaconnaissances, plans d'écriture et souvenirs d'expériences antérieures qu'il aura stockées et qui sont incontournables pour la réalisation de la tache demandée: "Connaissances sur le thème à traiter, mais aussi connaissances linguistiques et rhétoriques qui, toutes, seront actualisées lors du processus d'écriture" (HAYES; FLOWER, 1995, p. 49-72).

Fig. 1: Modèle Hayes et Flower (1995)

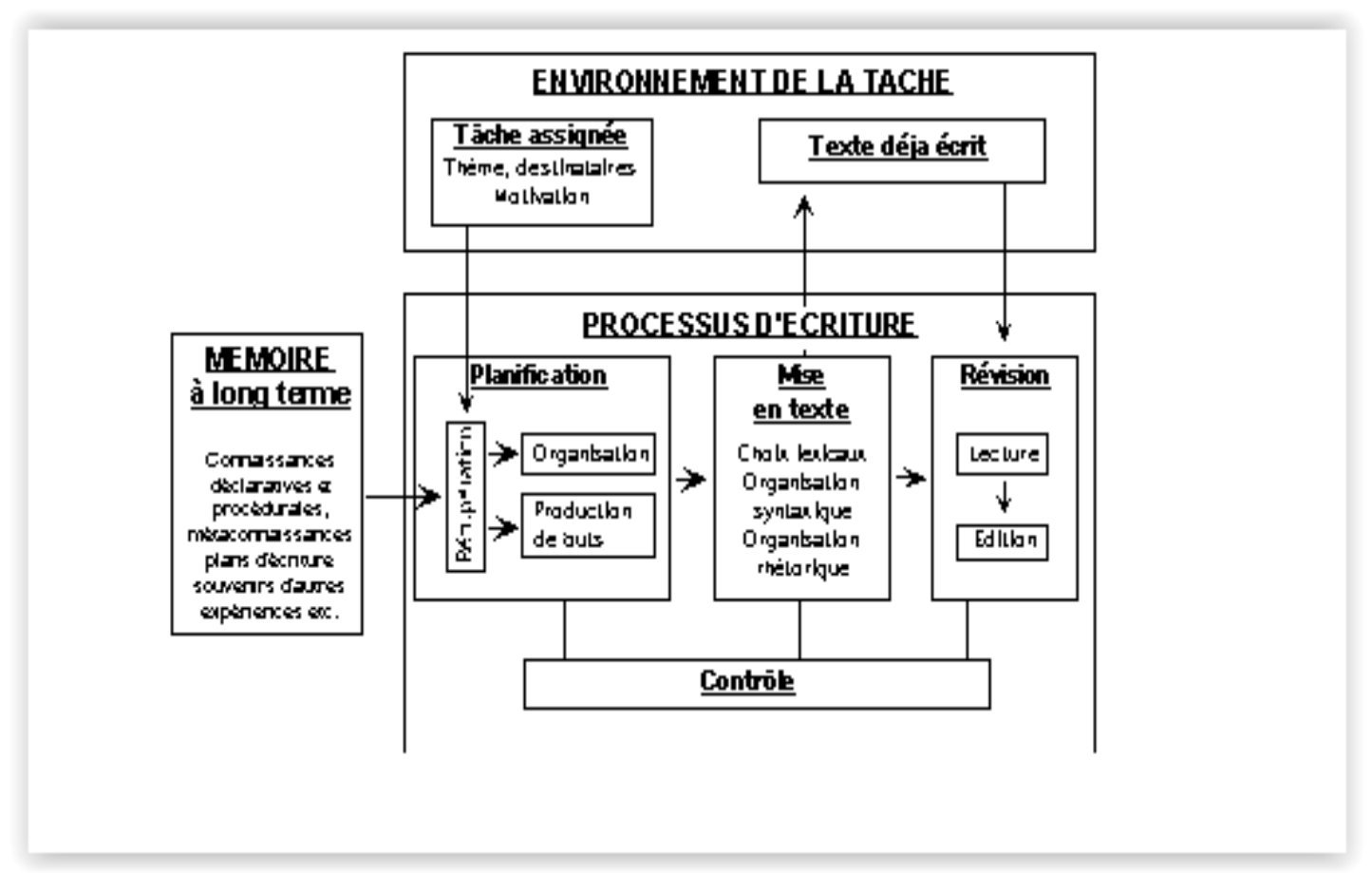

Secundo, nous évoquons les notions d'interdiscours et d'intertextualité caractéristiques de la production des énoncés, notamment ceux dits littéraires. Rappelons que:

L'interdiscours est au discours ce que l'intertexte est au texte [ Intertextualité] :- On peut appeler interdiscours un ensemble de discours (d'un même *champ discursif ou de champs distincts, d'époques différentes ...). Ainsi Courtine appelle-t-il interdiscours "une articulation contradictoire de *formations discursives référant à des formations idéologiques antagonistes " (1981 : 54). - Si l'on considère un discours particulier on peut aussi appeler interdiscours l'ensemble des unités discursives avec lesquelles il entre en relation. Selon le type de relation interdiscursive que l'on privilégie il pourra s'agir des discours cités, des discours antérieurs du même genre, des discours contemporains d'autres genres, etc. (MAINGUENEAU,1996, p. 50) 
Tierço, nous citons les propos de Dominique Maingueneau qui avance que l'intertextualité

renvoie tantôt à une propriété constitutive de tout texte, tantôt à l'ensemble des relations explicites ou implicites qu'un texte entretient avec d'autres textes. Dans la première acception il est une variante d'interdiscursivité. Mais si intertextualité et interdiscursivité ont un sens équivalent, ils ne lont cependant pas employés dans les mêmes domaines. C'est plutôt à propos de la littérature que l'on parle d'intertextualité, ou plus largement quand on a affaire à des 'textes au sens fort : les œuvres. (MAINGUENEAU, 1996, p. 51)

Finalement, ce travail trouve également son fondement dans les apports didactiques de Jean-Pierre Cuq et Isabelle Gruca (2005), qui proposent, en guise d'activités, d'écriture en production écrite ce qu'ils présentent comme matrices de textes. Eu égard à la réintégration des textes littéraires aux supports authentiques d'apprentissages, ces deux spécialistes de la didactique du français réclament les avantages des matrices textuelles qui optimisent la production d'un texte à partir de contraintes et / ou de canevas plus ou moins conséquent qui répond à des règles textuelles précises. Ainsi préconisent-ils à l'enseignant de s'inspirer d'un texte authentique:

II garde le canevas et les entrées de phrase: (mots thèmes, anaphores, articulateurs, ponctuation, etc), éventuellemlent le titre et les éléments paratextuels (s'ils existent et s'ils sont pertinents) s'il désire faire travailler sur le même thème. Les amorces peuvent se répéter ; à noter que, dans ce cas, l'activité peut être conduite avec des niveaux faibles ${ }^{1}$, voire même faux débutants. (CUQ; GRUCA, 2005, p. 453)

\section{LE CONTEXTE DE L’EXPÉRIENCE}

En tant qu'enseignant de FLS/FLE, j'ai mené cette expérience avec une classe de lettres et sciences humaines dans le cadre du premier module (voir Fig. 2) proposé pour les apprenants de la $1^{\text {ère }}$ année du baccalauréat pour l'année scolaire 2019-2020. II s'agit de projet pédagogique élaboré par les enseignants du français au sein du lycée. En effet, l'oeuvre en question doit faire l'objet d'étude sous formes de séquences articulées en quatre activités principales: lecture, langue, production orale et écrite.

\footnotetext{
${ }^{1}$ Les apprenants avec qui nous avons mené l'expérience représentent un échantillon d'élèves qui manifestent plusieurs lacunes et souffrent de difficultés conséquentes. (voir aperçu sur le rapport de l'évaluation diagnostique menée au début de l'année scolaire).
} 
Fig. 2: Module I du programme de $1^{\text {ère }}$ année de baccalauréat (MEN, 2007, p. 28)

\section{Progression de la première année du cycle du baccalauréat}

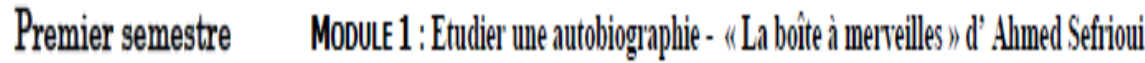

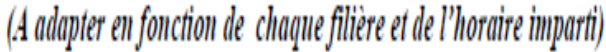

\begin{tabular}{|c|c|c|}
\hline Compétences & Activités & Contenus \\
\hline 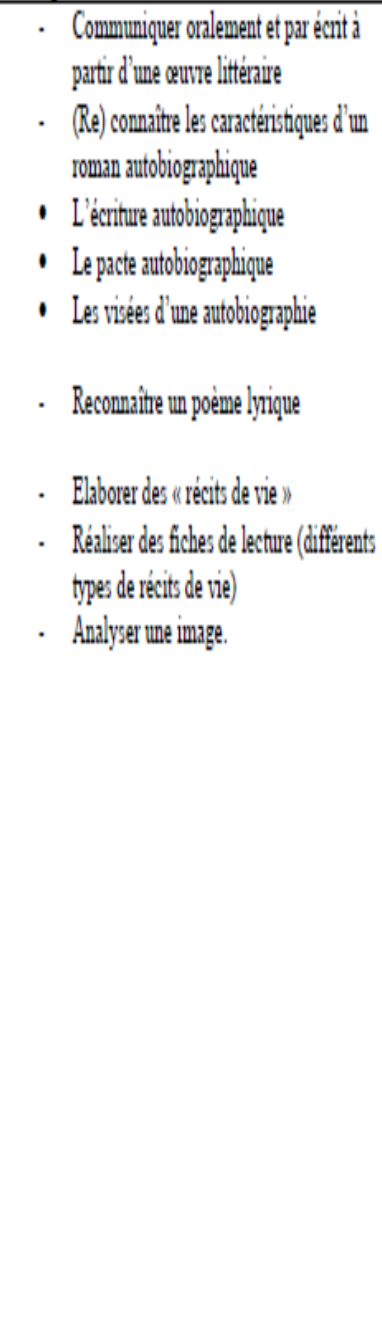 & 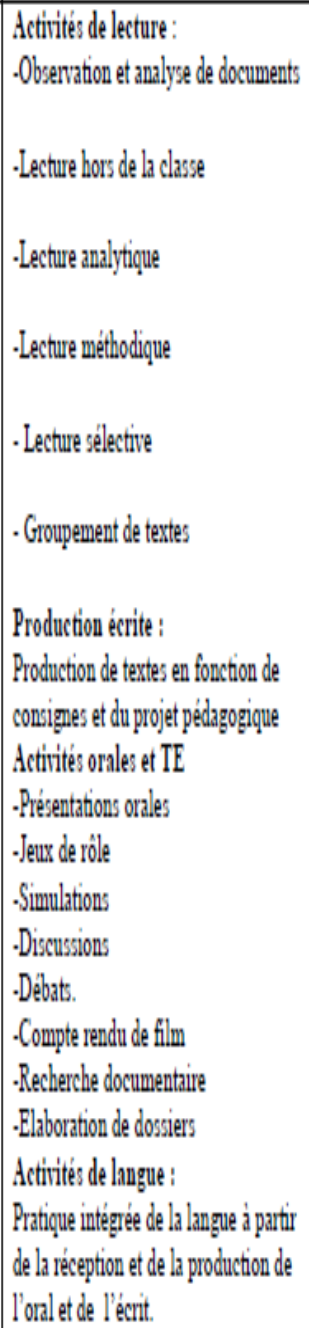 & 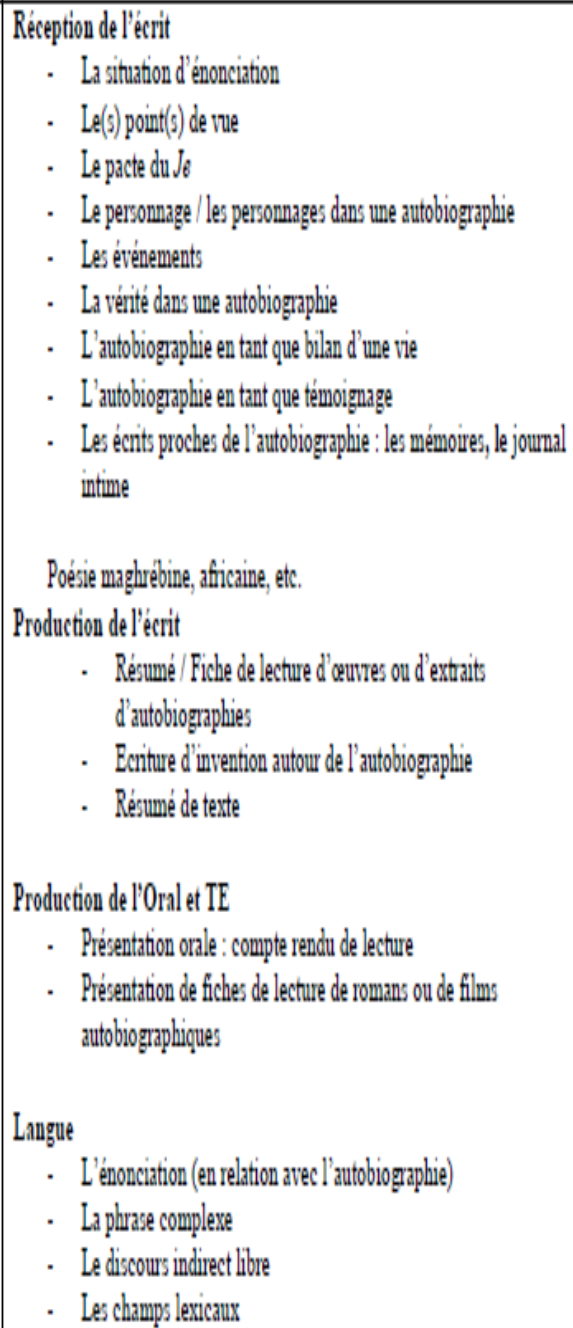 \\
\hline
\end{tabular}




\section{DESCRIPTION DE L’EXPÉRIENCE}

\section{PROFIL DE LA CLASSE-CIBLE}

Il convient avant de mettre en exergue le déroulement de l'expérience que la classe en question accumule plusieurs lacunes et difficultés comme le démontre le dépouillement des résultats de l'évaluation diagnostique effectuée au début de l'année. (Voir Fig. 3). En effet le sujet de production écrite proposée pour ce faire invitait les apprenants à produire le même genre et le même type de produit: autobiographique et narratif. Le libelle du sujet est le suivant: Vous aviez, vous aussi, tant désiré un objet que vous avez, enfin, acheté. Racontez votre expérience en décrivant vos sentiments après l'avoir acheté, dites ce que vous en avez fait, ce qu'il est advenu. (Nous avons veillé, expérience, d'appui, à expliquer le sujet et la consigne aux apprenants).

Fig. 3: tableau de dépouillement des notes de la première production écrite

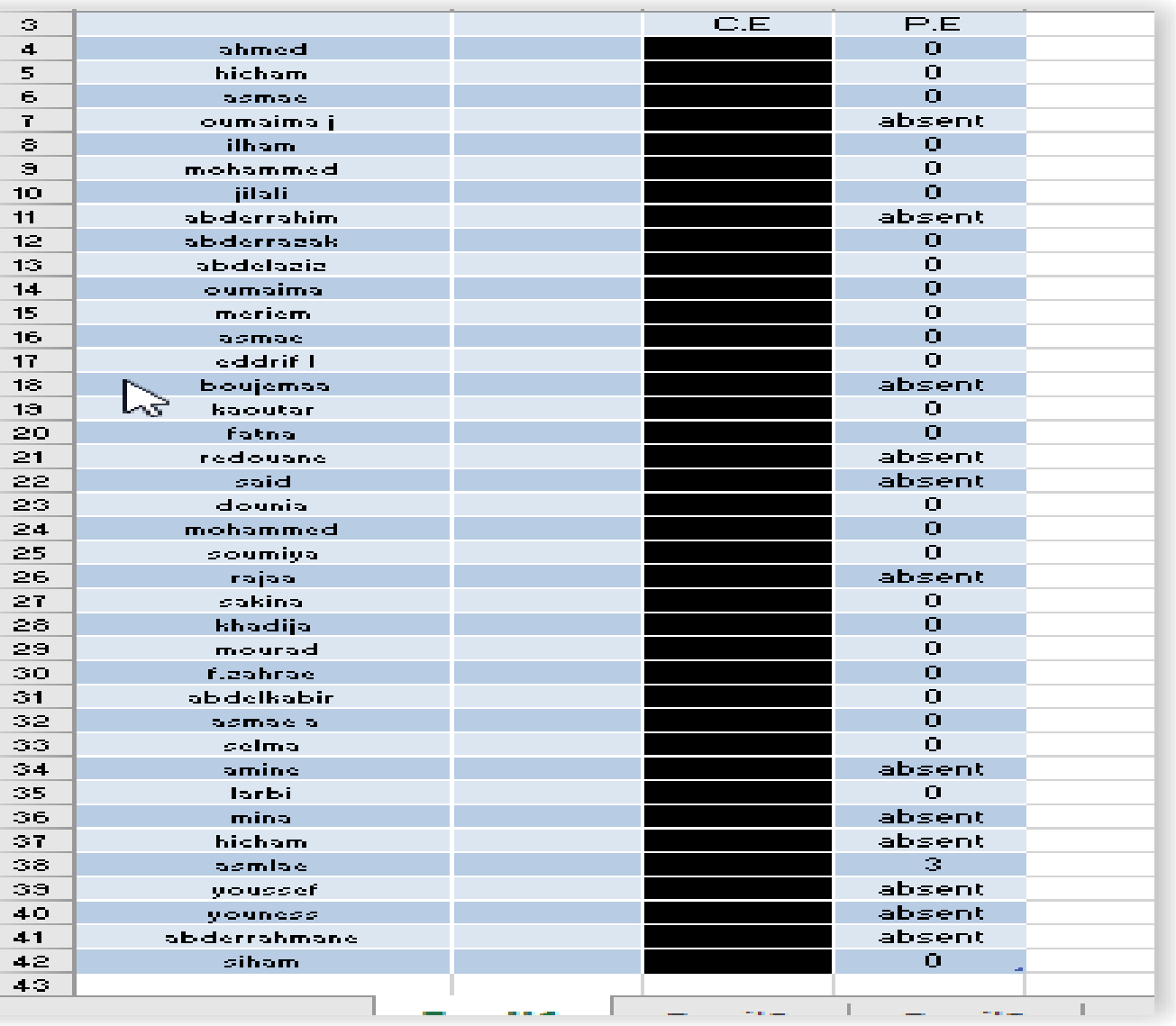


En guise d'annotation, le système d'évaluation au Maroc se base sur des notes numériques allant de 0/20 à 20/20 en fonction des réponses justes données par l'apprenant. Dans le tableau de dépouillement des résultats: la première colonne représente des prénoms fictifs des apprenants, la deuxième colonne quant à elle, contient les notes données pour la compétence de la compréhension de l'écrit et les outils de langue (figures de style, énonciation, champ lexical...) alors que la troisième colonne décline les notes obtenues en production écrite.

\section{DÉROULEMENT DU COURS/EXPÉRIENCE}

A cet égard, les apprenants, âgés entre 15 et 17 ans, ont été amenés à rédiger, dans le cadre de la première séquence du projet pédagogique de la littérature maghrébine d'expression française, et qui s'articule sur quatre activités: lecture, langue, production orale et production écrite, amenés à rédiger un texte de genre autobiographique en répondant au sujet et aux consignes qui figurent dans le corps de la fiche pédagogique suivante (Fig. 4):

Fig. 4: En-tête de la fiche pédagogique de l'activité de production écrite.

\begin{tabular}{|l|l|}
\hline Module 1 & Etudier un roman autobiographique \\
\hline Public & $1^{\text {ere }}$ année du cycle du baccalauréat \\
\hline Séquence & 1 \\
\hline Activité & Production écrite \\
\hline Support & Incipit de La Boite à Merveilles \\
\hline Compétence & Ecrire un texte autobiographique \\
\hline Capacités & $\begin{array}{l}\text {-Produire un texte en tenant compte des consignes } \\
\text {-Ecrire à la manière d'Ahmed Sefrioui (emploi du pronom } \\
\text { personnel “je"et des procédés énonciatifs corrélatifs) }\end{array}$ \\
\hline Démarche & $\begin{array}{l}\text { Cours dialogué } \\
\text { Travail en binôme } \\
\text { Correction collective }\end{array}$ \\
\hline $\begin{array}{l}\text { Sujet de de } \\
\text { rédaction }\end{array}$ & $\begin{array}{l}\text { A la manière d'Ahmed SEFRIOUI, rédigez l'incipit de ton récit } \\
\text { de vie }\end{array}$ \\
\hline Consignes & $\begin{array}{l}\text {-utilisez la 1're personne du singulier (2 pts) } \\
\text {-produis des énoncés ancrés dans la situation d'énonciation et } \\
\text { d'autres qui sont coupés de la situation d'énonciation (8 pts) } \\
\text { - faites part de votre personnalité (5 pts) } \\
\text {-utilisez une langue soignée. (5 pts) }\end{array}$ \\
\hline
\end{tabular}




\section{LA PHASE ORALE}

- Présentation du sujet

- explication des consignes

- recherche des idées: au début de cette étape, les apprenants reprennent le même support textuel ayant fait objet de la première séance de lecture:

Le soir, quand tous dorment, les riches dans leurs chaudes couvertures, les pauvres sur les marches des boutiques ou sous les porches des palais, moi je ne dors pas. Je songe à ma solitude et j'en sens tout le poids. Ma solitude ne date pas d'hier.

Je vois, au fond d'une impasse que le soleil ne visite jamais, un petit garçon de six ans, dresser un piège pour attraper un moineau mais le moineau ne vient jamais. II désire tant ce petit moineau ! II ne le mangera pas, il ne le martyrisera pas. II veut en faire son compagnon. Les pieds nus, sur la terre humide, il court jusqu'au bout de la ruelle pour voir passer les ânes et revient s'asseoir sur le pas de la maison et attendre l'arrivée du moineau qui ne vient pas. Le soir, il rentre le cœur gros et les yeux rougis, balançant au bout de son petit bras, un piège en fil de cuivre.

Nous habitions Dar Chouafa, la maison de la voyante. Effectivement, au rez-de-chaussée, habitait une voyante de grande réputation. Des quartiers les plus éloignés, des femmes de toutes les conditions venaient la consulter. Elle était voyante et quelque peu sorcière. Adepte de la confrérie des Gnaouas (gens de Guinée) elle s'offrait, une fois par mois, une séance de musique et de danses nègres. Des nuages de benjoin emplissaient la maison et les crotales et les guimbris nous empêchaient de dormir, toute la nuit. (SEFRIOUI, 1954, p. 3)

Le texte étant déjà lu au début de la séquence pédagogique, les apprenant le relisent silencieusement et en définissent le genre, la forme du discours, les temps verbaux, ainsi que la structure de séquence narrative, les lieux et temps désignés ainsi que les traits de personnalité. Le résultat de cette collecte d'informations et le suivant (Fig. 5):

Fig. 5: Collecte des informations

Genre d'écrit : autobiographique

Forme de discours : narratif

Temps verbaux : - temps du récit (énoncé coupé)

Présent d'énonciation

Structure de la séquence narrative :

- le présent du narrateur et son état de solitude « le soir, quand tous...ne date pas d'hier »

- le retour en arrière (flash-back) : « je vois au fond d'une impasse, un enfant » 


\section{LA PHASE ÉCRITE}

Nous avons demandé aux apprenants de rédiger individuellement et en binômes en respectant les consignes, tout comme dans l'évaluation diagnostiques, nous avons éludé de donner de l'aide aux apprenants.

\section{RÉSULTATS DE L'EXPÉRIENCE}

Pour l'ensemble des apprenants de cette classe, la correction des copies démontre un progrès considérable traduisible sur le tableau de dépouillement des notes obtenues ci-après (Fig. 6). Déjà la disparition de la valeur 0 qui dominait le premier tableau (Fig.3) dit long sur l'amélioration des acquis des apprenants en termes d'énonciation propre au genre autobiographique.

Fig. 6: Tableau de dépouillement des notes obtenues en production écrite après la correction des copies.

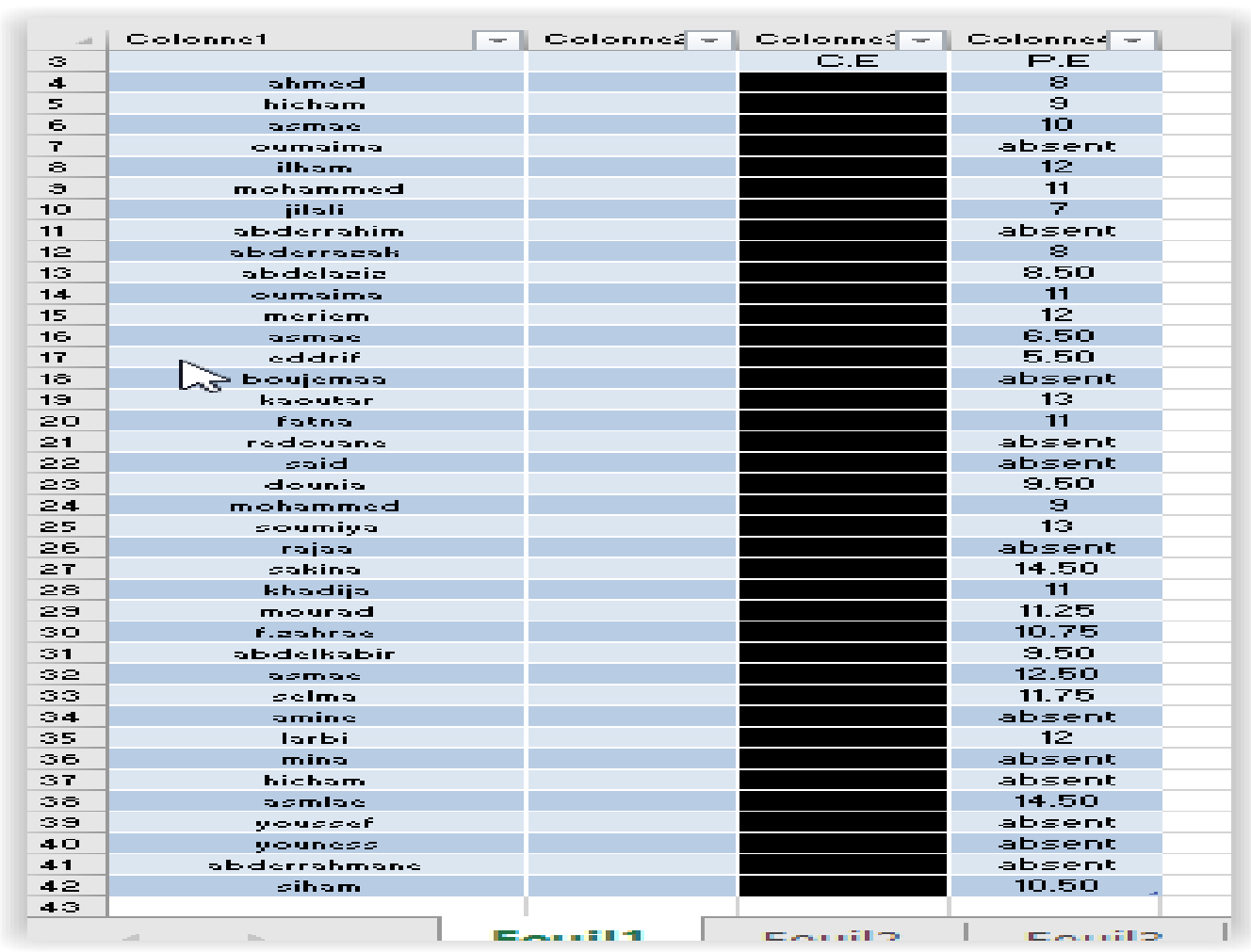


Nous allons transcrire ci-après, trois productions choisies aléatoirement (celle correspondant aux élèves portant les numéros 8,16 et 27 qui sont en difficulté, dont les notes au moment du diagnostic sont respectivement $0 / 20,0 / 20$ et $0 / 20$ et ce sans avoir corrigé les fautes orthographiques, syntaxiques ainsi que celles d'expression.

Texte 1 de l'élève $n$ 8:

Le soir, les autres parlent avec leurs parents et raconte les souvenirs dans le jour scolaire et moi je ne parle pas. Je pense à ma solitude et à ma tristesse. Les autres de mon âge il est très heureux va à la mer, la foret, le jardin des animaux et moi seul. Avant, j'étais une fille heureuse et j'aimais les voyages et les voisines qui habitaient dans le même village.

Texte 2 de l'élève $n$ 16:

La nuit, quand tous dorment, je seul à ma chambre, je songe à ma souvenir d'enfance quelques minut, je vois un petite fille de sept ans mais il y a un problem. Cette fille cria et pleuré parce que son père na pas acheté son cadeau de « achora " et quant je approchais à lui ...

Texte 3 de l'élève $n 27$ :

La nuit, quand tous ma famille dort, mes frères, et mes parents, la maison dans un silence et une obscurité, moi je ne dors pas, je réfléchis à l'examen. J'ouvre la fenêtre, soudain je regarde une petite fille de huit ans dans le champs pleurer et crier...

\section{DISCUSSION DES RÉSULTATS}

Nous constatons que les trois apprenantes confondues ont réussi à produire des textes qui répondent aux consignes se rapportant au genre et au types. Elles ont réinvesti la structure du texte de départ qui constitue un élément de la mémoire (modèle Hayes et Flower) en commençant par évoquer leur présent et en terminant par un retour en arrière sur leurs passés respectifs. En outre des expressions telles que: le soir (déictique), la nuit, avant...font des charnières chronologiques. Par ailleurs, les élèves $n 16$ et 27 ont réemployé la technique narrative et les constituants 
employés par l'auteur du texte authentique, par conséquent, réemploi du canevas, prôné par Cuq et Gruca (2005), déjà utilisé par le narrateur du texte-support: , «je vois un petite fille de sept ans »(élève n.8) , "je regarde une petite fille de huit ans » (élève n.27).

Les erreurs observées en matière de grammaire sont les manifestations flagrantes de lacunes accumulées chez les apprenants en particulier, et en général auprès du reste des apprenants de la classe.

\section{CONCLUSION}

Dans cette expérience, les apprenants de la classe ont fait preuve d'amélioration de leurs niveaux productifs en production écrite. Ce sont alors des apprenants à même de rédiger si les professeurs s'ouvrent sur différentes activités et méthodes et acceptent d'intégrer le texte littéraire dans leurs pratiques quotidiennes, celui-ci servant de modèle optimal pour l'apprentissage des outils et des concepts sur les genres et les types d'une part. D'autre part, à manifester les possibilités intertextuelles permettant aux apprenants d'appréhender les spécificités discursives des textes littéraires et aux enseignants de venir en aide à leurs apprenants. Nous invitons donc les enseignants du français à vulgariser à leurs apprenants la notion d'intertextualité tout en proposant des canevas de texte authentiques pouvant intriguer les intriguer de façon à les faire motiver pour la rédaction de produits de différents genres, tout en veillant à ce que les textes proposés fassent parties intégrantes des programmes et des curricula. Les difficultés observées chez les apprenants à la suite des opérations de l'évaluation diagnostique ne doivent pas représenter des écueils pour l'expérimentation de méthodes variées et modulées à l'aune des spécificités didactiques et pédagogiques des classes prises en charge.

\section{RÉFÉRENCES}

CUQ, J.-P.; GRUCA, I. Cours de didactique du français langue étrangère et seconde. Grenoble: Presses universitaires de Grenobles, 2005

DE BEAUGRANDE, R.-A. Linguistics as discourse: a case study from semantics. Word, New York, v. 35, ed. 1, p. 15-57, 20 abr. 1984 
DELL, G. S. The retrieval of phonological forms in production: Test of predictions from a connectionist model. Journal of Memory and Language, [s. I.], v. 27, p. 124142, 20 abr. 1988.

ELLIS, A. W. Normal writing processes and peripheral acquired dysgraphias. Language and cognitive processes, [s. I.], v. 3, n. 2, p. 99-127, 23 fev. 1988.

GARETT, M. Processes in language production. Linguistics: The Cambridge Survey, Language: Psychological and Biological Aspects, [s. I.], v. 3, p. 69-96, 21 abr. 1988.

GENETTE, G. La littérature au second degré. Paris: Seuil, 1982.

HARLEY, K. J. Symbolic connectionism: Toward third-generation theories of expertise. In: ERICSSON, K. A.; SMITH, J. Toward a general theory of expertise: Prospects and limits. Cambridge; New York: K. Anders Ericsson and Jacqui Smith, 1991. p. 301-335.

HARLEY, T. A. A critique of top-down independent levels of speech production: Evidence from non-plan-internal speech errors. Cognitive Science, [s. I.], v. 8, n. 3, p. 191-219, 23 jun. 1984.

HAYES, J. R. et al. Un nouveau modèle du processus d'écriture. In: BOYER, J.-Y. et al. (éds.). La production de textes: Vers un modèle d'enseignement de l'écriture. Montréal: Les Editions Logiques, 1995. p. 49-72.

HOLYOAK, K. J. Symbolic connectionism: Toward third-generation theories of expertise. In: ERICSSON, K. A.; SMITH, J. Toward a general theory of expertise: Prospects and limits. Cambridge: Cambridge University Press, 1991. p. 301-335.

LEVELT, W. J. M. Accessing words in speech production: Stages of processing and representations. Cognition, [s. I.], v. 42, p. 1-22, 1 mar. 1992.

MAINGUENEAU, D. Les termes clés de l'analyse du discours. 3. ed. Paris: Seuil, 1996.

MARGOLIN, D. I. The neuropsychology of writing and spelling: Semantic, phonological, motor, and perceptual processes. The quarterly journal of experimental psychology, [s. I.], v. 36, n. 3, p. 459-489, 23 out. 1984.

MARTLEW, M. The development of writing: Communication and cognition. In: WRITING in focus, [S. l.: s. n.], p. 257-276, 1983.

MINISTÈRE D'EDUCATION NATIONALE (MAROC). Direction des curricula. 2007. Orientations pédagogiques du français au cycle secondaire qualifiant, Rabat: Minstere d'éducation nationale, p. 6-32, 26 nov. 2019.

SEFRIOUI, A. La boîte à merveilles. Rabat: Les Écoles, 2006. 287 p.

VAN GALEN, G. P. Handwriting: Issues for a psychomotor theory. Human movement science, [s. I.], v. 10, n. 2, p. 165-191, 23 out. 1991. 


\section{L'auteur}

\section{Salah Tigourdi}

Salah Tigourdi a commencé son doctorat en 2016 au sein de la Faculté des Sciences de l'Education à l'Université Mohammed V à Rabat-Maroc Professeur de primaire depuis 2002, de français langue seconde au lycée d'Aljahid affilié à la Direction Provinciale de Safi au Maroc et enseignant vacataire de français langue étrangère à l'Alliance Française de Safi,.

${ }^{1}$ Salah Tigourdi Iniciou seu doutorado em 2016 na faculdade Ciências da Educação anexadas à Universidade Mohammed V em Rabat. Professor primário desde 2002, professor de francês como segunda língua na Aljahid High School, afiliado ao diretor provincial de Safi em Marrocos e professor de francês como língua estrangeira na Alliance Française de Safi, 